\title{
Benchmarking the completely renormalized equation-of-motion coupled-cluster approaches for vertical excitation energies (Supplemental Data)
}

\author{
Piotr Piecuch, ${ }^{1,2, *}$ Jared A. Hansen, ${ }^{1}$ and Adeayo O. Ajala ${ }^{1}$ \\ ${ }^{1}$ Department of Chemistry, Michigan State University, East Lansing, Michigan 48824, USA \\ ${ }^{2}$ Department of Physics and Astronomy, Michigan State University, East Lansing, Michigan 48824, USA
}

(Dated: July 17, 2015)

This supplemental data contains the information about the symmetry unique Cartesian coordinates of the groundstate geometries of the 28 molecules comprising the benchmark set proposed in Ref. [1] resulting from the MP2/6-31G* (Table I) and CR-CC(2,3),D/TZVP (Table II) optimizations.

[1] M. Schreiber, M. R. Silva-Junior, S. P. A. Sauer, and W. Thiel, J. Chem. Phys. 128, 134110 (2008).

${ }^{*}$ Corresponding author; e-mail: piecuch@chemistry.msu.edu. 
TABLE I: Symmetry unique Cartesian coordinates of the ground-state geometries for the 28 molecules comprising the benchmark set of Ref. [1] resulting from the MP2/6-31G* optimizations (taken from Ref. [1] and recalculated in this work).

\begin{tabular}{|c|c|c|c|c|}
\hline Molecule (symmetry) & Atom & $\mathrm{X}$ & $\mathrm{Y}$ & $\mathrm{Z}$ \\
\hline \multirow[t]{2}{*}{ Ethene $\left(D_{2 h}\right)$} & $\mathrm{H}$ & 0.000000 & 0.923274 & 1.238289 \\
\hline & $\mathrm{C}$ & 0.000000 & 0.000000 & 0.668188 \\
\hline \multirow[t]{5}{*}{ E-Butadiene $\left(\mathrm{C}_{2 h}\right)$} & $\mathrm{H}$ & -1.080977 & 2.558832 & 0.000000 \\
\hline & $\mathrm{H}$ & -2.103773 & 1.017723 & 0.000000 \\
\hline & $\mathrm{H}$ & 0.973565 & 1.219040 & 0.000000 \\
\hline & $\mathrm{C}$ & 0.000000 & -0.728881 & 0.000000 \\
\hline & $\mathrm{C}$ & -1.117962 & 1.474815 & 0.000000 \\
\hline \multirow[t]{7}{*}{ all-E-Hexatriene $\left(\mathrm{C}_{2 h}\right)$} & $\mathrm{H}$ & -0.953777 & 1.207691 & 0.000000 \\
\hline & $\mathrm{H}$ & 2.155816 & 0.952317 & 0.000000 \\
\hline & $\mathrm{H}$ & 2.125769 & 3.402692 & 0.000000 \\
\hline & $\mathrm{H}$ & 0.275642 & 3.397162 & 0.000000 \\
\hline & $\mathrm{C}$ & 0.000000 & 0.676808 & 0.000000 \\
\hline & $\mathrm{C}$ & 1.204938 & 1.485654 & 0.000000 \\
\hline & $\mathrm{C}$ & 1.203567 & 2.831663 & 0.000000 \\
\hline \multirow[t]{9}{*}{ all-E-Octatetraene $\left(\mathrm{C}_{2 h}\right)$} & $\mathrm{H}$ & 0.971328 & 1.220141 & 0.000000 \\
\hline & $\mathrm{H}$ & -2.098090 & 0.984719 & 0.000000 \\
\hline & $\mathrm{H}$ & -0.146884 & 3.418505 & 0.000000 \\
\hline & $\mathrm{H}$ & -2.193473 & 4.766086 & 0.000000 \\
\hline & $\mathrm{H}$ & -3.225698 & 3.230501 & 0.000000 \\
\hline & $\mathrm{C}$ & 0.000000 & 0.721498 & 0.000000 \\
\hline & $\mathrm{C}$ & 1.125020 & -1.479523 & 0.000000 \\
\hline & $\mathrm{C}$ & 1.121077 & -2.928812 & 0.000000 \\
\hline & $\mathrm{C}$ & 2.237388 & -3.682282 & 0.000000 \\
\hline \multirow[t]{4}{*}{ Cyclopropene $\left(\mathrm{C}_{2 v}\right)$} & $\mathrm{H}$ & 0.912650 & 0.000000 & 1.457504 \\
\hline & $\mathrm{H}$ & 0.000000 & -1.585659 & -1.038624 \\
\hline & $\mathrm{C}$ & 0.000000 & 0.000000 & 0.859492 \\
\hline & $\mathrm{C}$ & 0.000000 & -0.651229 & -0.499559 \\
\hline \multirow[t]{6}{*}{ Cyclopentadiene $\left(\mathrm{C}_{2 v}\right)$} & $\mathrm{H}$ & -0.879859 & 0.000000 & 1.874608 \\
\hline & $\mathrm{H}$ & 0.000000 & 2.211693 & 0.612518 \\
\hline & $\mathrm{H}$ & 0.000000 & 1.349811 & -1.886050 \\
\hline & $\mathrm{C}$ & 0.000000 & 0.000000 & 1.215652 \\
\hline & $\mathrm{C}$ & 0.000000 & -1.177731 & 0.285415 \\
\hline & $\mathrm{C}$ & 0.000000 & -0.732372 & -0.993420 \\
\hline \multirow[t]{6}{*}{ Norbornadiene $\left(\mathrm{C}_{2 v}\right)$} & $\mathrm{H}$ & 0.901419 & 0.000000 & 1.967823 \\
\hline & $\mathrm{H}$ & 0.000000 & 2.156504 & 0.616597 \\
\hline & $\mathrm{H}$ & 1.924341 & 1.340999 & -1.022814 \\
\hline & $\mathrm{C}$ & 0.000000 & 0.000000 & 1.346369 \\
\hline & $\mathrm{C}$ & 0.000000 & 1.119526 & 0.272221 \\
\hline & $\mathrm{C}$ & 1.235500 & 0.672374 & -0.517602 \\
\hline \multirow[t]{2}{*}{ Benzene $\left(D_{6 h}\right)$} & $\mathrm{H}$ & 2.151390 & 1.242106 & 0.000000 \\
\hline & $\mathrm{C}$ & 1.209657 & -0.698396 & 0.000000 \\
\hline \multirow[t]{5}{*}{ Naphthalene $\left(\mathrm{D}_{2 h}\right)$} & $\mathrm{H}$ & 1.240557 & 2.492735 & 0.000000 \\
\hline & $\mathrm{H}$ & 3.377213 & 1.246082 & 0.000000 \\
\hline & $\mathrm{C}$ & 0.000000 & 0.716253 & 0.000000 \\
\hline & $\mathrm{C}$ & 1.241539 & 1.403577 & 0.000000 \\
\hline & $\mathrm{C}$ & 2.432418 & 0.707325 & 0.000000 \\
\hline \multirow[t]{5}{*}{ Furan $\left(\mathrm{C}_{2 v}\right)$} & $\mathrm{H}$ & 0.000000 & 2.051058 & 0.851533 \\
\hline & $\mathrm{H}$ & 0.000000 & 1.371979 & -1.821224 \\
\hline & $\mathrm{C}$ & 0.000000 & 1.095840 & 0.348301 \\
\hline & $\mathrm{C}$ & 0.000000 & 0.714027 & -0.963274 \\
\hline & $\mathrm{O}$ & 0.000000 & 0.000000 & 1.164881 \\
\hline
\end{tabular}




\begin{tabular}{|c|c|c|c|c|}
\hline Molecule (symmetry) & Atom & $\mathrm{X}$ & $\mathrm{Y}$ & $\mathrm{Z}$ \\
\hline \multirow[t]{6}{*}{ Pyrrole $\left(\mathrm{C}_{2 v}\right)$} & $\mathrm{H}$ & 0.000000 & 2.114611 & 0.770889 \\
\hline & $\mathrm{H}$ & 0.000000 & 1.358585 & -1.850224 \\
\hline & $\mathrm{H}$ & 0.000000 & 0.000000 & 2.130670 \\
\hline & $\mathrm{C}$ & 0.000000 & 1.125828 & 0.333870 \\
\hline & $\mathrm{C}$ & 0.000000 & 0.709235 & -0.984789 \\
\hline & $\mathrm{N}$ & 0.000000 & 0.000000 & 1.119862 \\
\hline \multirow[t]{9}{*}{ Imidazole $\left(\mathrm{C}_{s}\right)$} & $\mathrm{H}$ & 0.000000 & 2.119822 & 0.714354 \\
\hline & $\mathrm{H}$ & 0.000000 & 1.202262 & -1.904898 \\
\hline & $\mathrm{H}$ & 0.000000 & -2.104815 & 0.663782 \\
\hline & $\mathrm{H}$ & 0.000000 & -0.010302 & 2.116597 \\
\hline & $\mathrm{C}$ & 0.000000 & 1.120107 & 0.305897 \\
\hline & $\mathrm{C}$ & 0.000000 & 0.635508 & -0.983749 \\
\hline & $\mathrm{C}$ & 0.000000 & -1.091835 & 0.283881 \\
\hline & $\mathrm{N}$ & 0.000000 & -0.741378 & -0.994001 \\
\hline & $\mathrm{N}$ & 0.000000 & 0.000000 & 1.104571 \\
\hline \multirow{7}{*}{ Pyridine $\left(\mathrm{C}_{2 v}\right)$} & $\mathrm{H}$ & 0.000000 & 2.061947 & 1.308539 \\
\hline & $\mathrm{H}$ & 0.000000 & 2.156804 & -1.184054 \\
\hline & $\mathrm{H}$ & 0.000000 & 0.000000 & -2.475074 \\
\hline & $\mathrm{C}$ & 0.000000 & 1.145417 & 0.721005 \\
\hline & $\mathrm{C}$ & 0.000000 & 1.197637 & -0.673735 \\
\hline & $\mathrm{C}$ & 0.000000 & 0.000000 & -1.387901 \\
\hline & $\mathrm{N}$ & 0.000000 & 0.000000 & 1.426610 \\
\hline \multirow[t]{3}{*}{ Pyrazine $\left(\mathrm{D}_{2 h}\right)$} & $\mathrm{H}$ & 0.000000 & 2.068464 & 1.258236 \\
\hline & $\mathrm{C}$ & 0.000000 & 1.135920 & 0.697884 \\
\hline & $\mathrm{N}$ & 0.000000 & 0.000000 & 1.417402 \\
\hline \multirow[t]{7}{*}{ Pyrimidine $\left(\mathrm{C}_{2 v}\right)$} & $\mathrm{H}$ & 0.000000 & 2.156588 & 1.120200 \\
\hline & $\mathrm{H}$ & 0.000000 & 0.000000 & -2.400385 \\
\hline & $\mathrm{H}$ & 0.000000 & 0.000000 & 2.440403 \\
\hline & $\mathrm{C}$ & 0.000000 & 1.186684 & 0.626213 \\
\hline & $\mathrm{C}$ & 0.000000 & 0.000000 & -1.312625 \\
\hline & $\mathrm{C}$ & 0.000000 & 0.000000 & 1.354949 \\
\hline & $\mathrm{N}$ & 0.000000 & 1.203523 & -0.717781 \\
\hline \multirow[t]{5}{*}{ Pyridazine $\left(\mathrm{C}_{2 v}\right)$} & $\mathrm{H}$ & 0.000000 & 2.409486 & -0.149325 \\
\hline & $\mathrm{H}$ & 0.000000 & 1.271234 & 2.102647 \\
\hline & $\mathrm{C}$ & 0.000000 & 1.325698 & -0.063084 \\
\hline & $\mathrm{C}$ & 0.000000 & 0.693095 & 1.182948 \\
\hline & $\mathrm{N}$ & 0.000000 & 0.674211 & -1.238929 \\
\hline \multirow[t]{6}{*}{ s-Triazine $\left(\mathrm{D}_{3 h}\right)$} & $\mathrm{H}$ & 0.000000 & 0.000000 & 2.386083 \\
\hline & $\mathrm{H}$ & 0.000000 & 2.066408 & -1.193041 \\
\hline & $\mathrm{C}$ & 0.000000 & 0.000000 & 1.298345 \\
\hline & $\mathrm{C}$ & 0.000000 & 1.124400 & -0.649173 \\
\hline & $\mathrm{N}$ & 0.000000 & 0.000000 & -1.379450 \\
\hline & $\mathrm{N}$ & 0.000000 & 1.194639 & 0.689726 \\
\hline \multirow[t]{3}{*}{ s-Tetrazine $\left(\mathrm{D}_{2 h}\right)$} & $\mathrm{H}$ & 0.000000 & 0.000000 & -2.354794 \\
\hline & $\mathrm{C}$ & 0.000000 & 0.000000 & 1.269044 \\
\hline & $\mathrm{N}$ & 0.000000 & 1.204572 & 0.670429 \\
\hline \multirow[t]{3}{*}{ Formaldehyde $\left(\mathrm{C}_{2 v}\right)$} & $\mathrm{H}$ & 0.000000 & 0.934473 & -0.588078 \\
\hline & $\mathrm{C}$ & 0.000000 & 0.000000 & 0.000000 \\
\hline & $\mathrm{O}$ & 0.000000 & 0.000000 & 1.221104 \\
\hline \multirow[t]{5}{*}{ Acetone $\left(\mathrm{C}_{2 v}\right)$} & $\mathrm{H}$ & 0.000000 & 2.136732 & -0.112445 \\
\hline & $\mathrm{H}$ & -0.881334 & 1.333733 & -1.443842 \\
\hline & $\mathrm{C}$ & 0.000000 & 0.000000 & 0.000000 \\
\hline & $\mathrm{C}$ & 0.000000 & 1.287253 & -0.795902 \\
\hline & $\mathrm{O}$ & 0.000000 & 0.000000 & 1.227600 \\
\hline
\end{tabular}




\begin{tabular}{|c|c|c|c|c|}
\hline Molecule (symmetry) & Atom & $\mathrm{X}$ & $\mathrm{Y}$ & $\mathrm{Z}$ \\
\hline \multirow[t]{4}{*}{ p-Benzoquinone $\left(\mathrm{D}_{2 h}\right)$} & $\mathrm{H}$ & 0.000000 & 2.182973 & 1.259286 \\
\hline & $\mathrm{C}$ & 0.000000 & 0.000000 & 1.441079 \\
\hline & $\mathrm{C}$ & 0.000000 & 1.266644 & 0.674582 \\
\hline & $\mathrm{O}$ & 0.000000 & 0.000000 & 2.678518 \\
\hline \multirow{6}{*}{ Formamide $\left(\mathrm{C}_{s}\right)$} & $\mathrm{H}$ & -0.927427 & -0.600301 & 0.000000 \\
\hline & $\mathrm{H}$ & 1.070498 & -1.782390 & 0.000000 \\
\hline & $\mathrm{H}$ & 2.024514 & -0.325050 & 0.000000 \\
\hline & $\mathrm{C}$ & 0.000000 & 0.000000 & 0.000000 \\
\hline & $\mathrm{O}$ & 0.000000 & 1.225060 & 0.000000 \\
\hline & $\mathrm{N}$ & 1.119392 & -0.775069 & 0.000000 \\
\hline \multirow[t]{8}{*}{ Acetamide $\left(\mathrm{C}_{s}\right)$} & $\mathrm{H}$ & 1.173209 & -1.735763 & 0.000000 \\
\hline & $\mathrm{H}$ & 2.035841 & -0.226201 & 0.000000 \\
\hline & $\mathrm{H}$ & -2.121189 & -0.156089 & 0.000000 \\
\hline & $\mathrm{H}$ & -1.310647 & -1.472742 & 0.885504 \\
\hline & $\mathrm{C}$ & 0.000000 & 0.000000 & 0.000000 \\
\hline & $\mathrm{C}$ & -1.267042 & -0.831610 & 0.000000 \\
\hline & $\mathrm{O}$ & 0.000000 & 1.229439 & 0.000000 \\
\hline & $\mathrm{N}$ & 1.158967 & -0.727718 & 0.000000 \\
\hline \multirow[t]{10}{*}{ Propanamide $\left(\mathrm{C}_{s}\right)$} & $\mathrm{H}$ & 1.171887 & -1.734653 & 0.000000 \\
\hline & $\mathrm{H}$ & 2.036508 & -0.225526 & 0.000000 \\
\hline & $\mathrm{H}$ & -1.256737 & -1.492368 & 0.877197 \\
\hline & $\mathrm{H}$ & -3.420939 & -0.590421 & 0.000000 \\
\hline & $\mathrm{H}$ & -2.544313 & 0.678541 & -0.880209 \\
\hline & $\mathrm{C}$ & 0.000000 & 0.000000 & 0.000000 \\
\hline & $\mathrm{C}$ & -1.272727 & -0.833216 & 0.000000 \\
\hline & $\mathrm{C}$ & -2.523376 & 0.033790 & 0.000000 \\
\hline & $\mathrm{O}$ & 0.000000 & 1.230373 & 0.000000 \\
\hline & $\mathrm{N}$ & 1.159100 & -0.726409 & 0.000000 \\
\hline \multirow[t]{13}{*}{ Cytosine $\left(\mathrm{C}_{s}\right)$} & $\mathrm{H}$ & -2.114860 & -1.429678 & 0.000000 \\
\hline & $\mathrm{H}$ & -0.173973 & -2.806186 & 0.000000 \\
\hline & $\mathrm{H}$ & 2.073228 & -1.658021 & 0.000000 \\
\hline & $\mathrm{H}$ & 3.175240 & 0.564335 & 0.000000 \\
\hline & $\mathrm{H}$ & 2.235202 & 2.033636 & 0.000000 \\
\hline & $\mathrm{C}$ & -0.060783 & -1.726152 & 0.000000 \\
\hline & $\mathrm{C}$ & 1.144884 & -1.099470 & 0.000000 \\
\hline & $\mathrm{C}$ & 1.107049 & 0.338190 & 0.000000 \\
\hline & $\mathrm{C}$ & -1.227573 & 0.430359 & 0.000000 \\
\hline & $\mathrm{O}$ & -2.315109 & 0.998271 & 0.000000 \\
\hline & $\mathrm{N}$ & 0.000000 & 1.058130 & 0.000000 \\
\hline & $\mathrm{N}$ & -1.201178 & -0.989148 & 0.000000 \\
\hline & $\mathrm{N}$ & 2.278974 & 1.024187 & 0.000000 \\
\hline \multirow[t]{14}{*}{ Thymine $\left(\mathrm{C}_{s}\right)$} & $\mathrm{H}$ & 0.217481 & -2.676720 & 0.000000 \\
\hline & $\mathrm{H}$ & 2.052694 & 0.924773 & 0.000000 \\
\hline & $\mathrm{H}$ & -1.943101 & -1.709021 & 0.000000 \\
\hline & $\mathrm{H}$ & -3.360610 & 0.309754 & 0.000000 \\
\hline & $\mathrm{H}$ & -2.616463 & 1.665008 & 0.879105 \\
\hline & $\mathrm{C}$ & 1.356951 & -0.994496 & 0.000000 \\
\hline & $\mathrm{C}$ & 0.000000 & 1.121102 & 0.000000 \\
\hline & $\mathrm{C}$ & -1.214538 & 0.306431 & 0.000000 \\
\hline & $\mathrm{C}$ & -1.085764 & -1.041812 & 0.000000 \\
\hline & $\mathrm{C}$ & -2.529824 & 1.020445 & 0.000000 \\
\hline & $\mathrm{O}$ & 2.444132 & -1.558490 & 0.000000 \\
\hline & $\mathrm{O}$ & 0.023681 & 2.350992 & 0.000000 \\
\hline & $\mathrm{N}$ & 0.145112 & -1.666249 & 0.000000 \\
\hline & $\mathrm{N}$ & 1.192460 & 0.382130 & 0.000000 \\
\hline \multirow[t]{3}{*}{ Uracil $\left(\mathrm{C}_{s}\right)$} & $\mathrm{H}$ & -2.025413 & -1.517742 & 0.000000 \\
\hline & $\mathrm{H}$ & -0.021861 & 1.995767 & 0.000000 \\
\hline & $\mathrm{H}$ & 2.182391 & -1.602586 & 0.000000 \\
\hline
\end{tabular}




\begin{tabular}{|c|c|c|c|c|}
\hline Molecule (symmetry) & Atom & $\mathrm{X}$ & $\mathrm{Y}$ & $\mathrm{Z}$ \\
\hline & $\mathrm{H}$ & -0.026659 & -2.791719 & 0.000000 \\
\hline & $\mathrm{C}$ & -1.239290 & 0.359825 & 0.000000 \\
\hline & $\mathrm{C}$ & 1.279718 & 0.392094 & 0.000000 \\
\hline & $\mathrm{C}$ & 1.243729 & -1.064577 & 0.000000 \\
\hline & $\mathrm{C}$ & 0.055755 & -1.709579 & 0.000000 \\
\hline & $\mathrm{O}$ & -2.308803 & 0.954763 & 0.000000 \\
\hline & $\mathrm{O}$ & 2.287387 & 1.092936 & 0.000000 \\
\hline & $\mathrm{N}$ & -1.139515 & -1.026364 & 0.000000 \\
\hline & $\mathrm{N}$ & 0.000000 & 0.978951 & 0.000000 \\
\hline \multirow[t]{15}{*}{ Adenine $\left(\mathrm{C}_{s}\right)$} & $\mathrm{H}$ & 0.974930 & -3.075149 & 0.000000 \\
\hline & $\mathrm{H}$ & 2.134658 & 2.075802 & 0.000000 \\
\hline & $\mathrm{H}$ & 3.312010 & 0.776987 & 0.000000 \\
\hline & $\mathrm{H}$ & -3.052077 & -0.334232 & 0.000000 \\
\hline & $\mathrm{H}$ & -2.711876 & 2.203052 & 0.000000 \\
\hline & $\mathrm{C}$ & 0.662834 & -2.032900 & 0.000000 \\
\hline & $\mathrm{C}$ & 1.359313 & 0.172553 & 0.000000 \\
\hline & $\mathrm{C}$ & 0.000000 & 0.547434 & 0.000000 \\
\hline & $\mathrm{C}$ & -0.924835 & -0.500714 & 0.000000 \\
\hline & $\mathrm{C}$ & -1.906806 & 1.478795 & 0.000000 \\
\hline & $\mathrm{N}$ & -0.658577 & -1.817838 & 0.000000 \\
\hline & $\mathrm{N}$ & 1.672594 & -1.133202 & 0.000000 \\
\hline & $\mathrm{N}$ & -2.150759 & 0.128726 & 0.000000 \\
\hline & $\mathrm{N}$ & -0.616118 & 1.783396 & 0.000000 \\
\hline & $\mathrm{N}$ & 2.352763 & 1.090709 & 0.000000 \\
\hline
\end{tabular}


TABLE II: Symmetry unique Cartesian coordinates of the ground-state geometries of the 28 molecules comprising the benchmark set of Ref. [1] resulting from the CR-CC(2,3),D/TZVP optimizations carried out in this work.

\begin{tabular}{|c|c|c|c|c|}
\hline Molecule (symmetry) & Atom & $\mathrm{X}$ & $\mathrm{Y}$ & $\mathrm{Z}$ \\
\hline \multirow[t]{2}{*}{ Ethene $\left(\mathrm{D}_{2 h}\right)$} & $\mathrm{H}$ & 0.000000 & 0.924035 & 1.236513 \\
\hline & $\mathrm{C}$ & 0.000000 & 0.000000 & 0.668881 \\
\hline \multirow[t]{5}{*}{ E-Butadiene $\left(\mathrm{C}_{2 h}\right)$} & $\mathrm{H}$ & -2.733502 & 0.488401 & 0.000000 \\
\hline & $\mathrm{H}$ & -1.996346 & -1.209626 & 0.000000 \\
\hline & $\mathrm{H}$ & -0.493204 & 1.480741 & 0.000000 \\
\hline & $\mathrm{C}$ & 0.613255 & -0.399134 & 0.000000 \\
\hline & $\mathrm{C}$ & -1.846790 & -0.134268 & 0.000000 \\
\hline \multirow[t]{7}{*}{ all-E-Hexatriene $\left(\mathrm{C}_{2 h}\right)$} & $\mathrm{H}$ & -0.689671 & -1.373825 & 0.000000 \\
\hline & $\mathrm{H}$ & -1.779265 & 1.550035 & 0.000000 \\
\hline & $\mathrm{H}$ & -3.983818 & 0.481440 & 0.000000 \\
\hline & $\mathrm{H}$ & -3.188581 & -1.190249 & 0.000000 \\
\hline & $\mathrm{C}$ & -0.611506 & -0.287351 & 0.000000 \\
\hline & $\mathrm{C}$ & -1.860731 & 0.465059 & 0.000000 \\
\hline & $\mathrm{C}$ & -3.076386 & -0.110589 & 0.000000 \\
\hline \multirow[t]{9}{*}{ all-E-Octatetraene $\left(\mathrm{C}_{2 h}\right)$} & $\mathrm{H}$ & -0.609354 & 1.435522 & 0.000000 \\
\hline & $\mathrm{H}$ & -1.864325 & -1.371454 & 0.000000 \\
\hline & $\mathrm{H}$ & -3.082792 & 1.500066 & 0.000000 \\
\hline & $\mathrm{H}$ & -5.238862 & 0.334070 & 0.000000 \\
\hline & $\mathrm{H}$ & -4.370232 & -1.299431 & 0.000000 \\
\hline & $\mathrm{C}$ & -0.638128 & 0.346740 & 0.000000 \\
\hline & $\mathrm{C}$ & 1.835457 & 0.282916 & 0.000000 \\
\hline & $\mathrm{C}$ & 3.116293 & -0.412631 & 0.000000 \\
\hline & $\mathrm{C}$ & 4.305668 & 0.215978 & 0.000000 \\
\hline \multirow[t]{4}{*}{ Cyclopropene $\left(\mathrm{C}_{2 v}\right)$} & $\mathrm{H}$ & 0.914476 & 0.000000 & 1.478608 \\
\hline & $\mathrm{H}$ & 0.000000 & -1.579439 & -1.022564 \\
\hline & $\mathrm{C}$ & 0.000000 & 0.000000 & 0.887272 \\
\hline & $\mathrm{C}$ & 0.000000 & -0.650147 & -0.480853 \\
\hline \multirow[t]{6}{*}{ Cyclopentadiene $\left(\mathrm{C}_{2 v}\right)$} & $\mathrm{H}$ & -0.884693 & 0.000000 & 1.884064 \\
\hline & $\mathrm{H}$ & 0.000000 & 2.210781 & 0.627891 \\
\hline & $\mathrm{H}$ & 0.000000 & 1.348160 & -1.876762 \\
\hline & $\mathrm{C}$ & 0.000000 & 0.000000 & 1.235311 \\
\hline & $\mathrm{C}$ & 0.000000 & -1.181911 & 0.294218 \\
\hline & $\mathrm{C}$ & 0.000000 & -0.737921 & -0.982749 \\
\hline \multirow[t]{6}{*}{ Norbornadiene $\left(\mathrm{C}_{2 v}\right)$} & $\mathrm{H}$ & 0.901246 & 0.000000 & 1.976906 \\
\hline & $\mathrm{H}$ & 0.000000 & 2.156931 & 0.625501 \\
\hline & $\mathrm{H}$ & 1.933914 & 1.340614 & -1.010326 \\
\hline & $\mathrm{C}$ & 0.000000 & 0.000000 & 1.357782 \\
\hline & $\mathrm{C}$ & 0.000000 & 1.123491 & 0.279727 \\
\hline & $\mathrm{C}$ & 1.244064 & 0.671585 & -0.512920 \\
\hline \multirow[t]{2}{*}{ Benzene $\left(\mathrm{D}_{6 h}\right)$} & $\mathrm{H}$ & 0.000000 & 2.482672 & 0.000000 \\
\hline & $\mathrm{C}$ & 0.000000 & 1.397828 & 0.000000 \\
\hline \multirow[t]{5}{*}{ Naphthalene $\left(\mathrm{D}_{2 h}\right)$} & $\mathrm{H}$ & 1.241938 & 2.490352 & 0.000000 \\
\hline & $\mathrm{H}$ & 3.377709 & 1.245631 & 0.000000 \\
\hline & $\mathrm{C}$ & 0.000000 & 0.712349 & 0.000000 \\
\hline & $\mathrm{C}$ & 1.245787 & 1.404252 & 0.000000 \\
\hline & $\mathrm{C}$ & 2.434128 & 0.710577 & 0.000000 \\
\hline \multirow[t]{5}{*}{ Furan $\left(\mathrm{C}_{2 v}\right)$} & $\mathrm{H}$ & 0.000000 & 2.050655 & 0.816080 \\
\hline & $\mathrm{H}$ & 0.000000 & 1.377422 & -1.844443 \\
\hline & $\mathrm{C}$ & 0.000000 & 1.095512 & 0.318614 \\
\hline & $\mathrm{C}$ & 0.000000 & 0.720764 & -0.989032 \\
\hline & $\mathrm{O}$ & 0.000000 & 0.000000 & 1.138748 \\
\hline
\end{tabular}




\begin{tabular}{|c|c|c|c|c|}
\hline Molecule (symmetry) & Atom & $\mathrm{X}$ & $\mathrm{Y}$ & $\mathrm{Z}$ \\
\hline \multirow[t]{6}{*}{ Pyrrole $\left(\mathrm{C}_{2 v}\right)$} & $\mathrm{H}$ & 0.000000 & 2.113533 & 0.765168 \\
\hline & $\mathrm{H}$ & 0.000000 & 1.361742 & -1.848823 \\
\hline & $\mathrm{H}$ & 0.000000 & 0.000000 & 2.129579 \\
\hline & $\mathrm{C}$ & 0.000000 & 1.126599 & 0.330605 \\
\hline & $\mathrm{C}$ & 0.000000 & 0.714934 & -0.985073 \\
\hline & $\mathrm{N}$ & 0.000000 & 0.000000 & 1.123459 \\
\hline \multirow[t]{9}{*}{ Imidazole $\left(\mathrm{C}_{s}\right)$} & $\mathrm{H}$ & -1.619793 & 1.573819 & 0.000000 \\
\hline & $\mathrm{H}$ & 1.123222 & 1.935864 & 0.000000 \\
\hline & $\mathrm{H}$ & 0.334319 & -2.168241 & 0.000000 \\
\hline & $\mathrm{H}$ & -1.909946 & -0.965998 & 0.000000 \\
\hline & $\mathrm{C}$ & -0.806312 & 0.867796 & 0.000000 \\
\hline & $\mathrm{C}$ & 0.557098 & 1.018433 & 0.000000 \\
\hline & $\mathrm{C}$ & 0.213535 & -1.095313 & 0.000000 \\
\hline & $\mathrm{N}$ & 1.191626 & -0.213966 & 0.000000 \\
\hline & $\mathrm{N}$ & -1.017600 & -0.499248 & 0.000000 \\
\hline \multirow[t]{7}{*}{ Pyridine $\left(\mathrm{C}_{2 v}\right)$} & $\mathrm{H}$ & 0.000000 & 2.057742 & 1.279370 \\
\hline & $\mathrm{H}$ & 0.000000 & 2.157421 & -1.204811 \\
\hline & $\mathrm{H}$ & 0.000000 & 0.000000 & -2.499000 \\
\hline & $\mathrm{C}$ & 0.000000 & 1.142017 & 0.696089 \\
\hline & $\mathrm{C}$ & 0.000000 & 1.198401 & -0.699905 \\
\hline & $\mathrm{C}$ & 0.000000 & 0.000000 & -1.414468 \\
\hline & $\mathrm{N}$ & 0.000000 & 0.000000 & 1.403713 \\
\hline \multirow[t]{3}{*}{ Pyrazine $\left(\mathrm{D}_{2 h}\right)$} & $\mathrm{H}$ & 0.000000 & 2.064971 & 1.251200 \\
\hline & $\mathrm{C}$ & 0.000000 & 1.131290 & 0.698767 \\
\hline & $\mathrm{N}$ & 0.000000 & 0.000000 & 1.419992 \\
\hline \multirow[t]{7}{*}{ Pyrimidine $\left(\mathrm{C}_{2 v}\right)$} & $\mathrm{H}$ & 0.000000 & 2.151421 & 1.143883 \\
\hline & $\mathrm{H}$ & 0.000000 & 0.000000 & -2.367202 \\
\hline & $\mathrm{H}$ & 0.000000 & 0.000000 & 2.465179 \\
\hline & $\mathrm{C}$ & 0.000000 & 1.185180 & 0.649436 \\
\hline & $\mathrm{C}$ & 0.000000 & 0.000000 & -1.282724 \\
\hline & $\mathrm{C}$ & 0.000000 & 0.000000 & 1.382204 \\
\hline & $\mathrm{N}$ & 0.000000 & 1.204114 & -0.692499 \\
\hline \multirow[t]{5}{*}{ Pyridazine $\left(\mathrm{C}_{2 v}\right)$} & $\mathrm{H}$ & 0.000000 & 2.402231 & -0.101371 \\
\hline & $\mathrm{H}$ & 0.000000 & 1.274904 & 2.147804 \\
\hline & $\mathrm{C}$ & 0.000000 & 1.321686 & -0.017415 \\
\hline & $\mathrm{C}$ & 0.000000 & 0.691188 & 1.234582 \\
\hline & $\mathrm{N}$ & 0.000000 & 0.673098 & -1.186891 \\
\hline \multirow[t]{6}{*}{ s-Triazine $\left(\mathrm{D}_{3 h}\right)$} & $\mathrm{H}$ & 0.000000 & 0.000000 & 2.379949 \\
\hline & $\mathrm{H}$ & 0.000000 & -2.061096 & -1.189974 \\
\hline & $\mathrm{C}$ & 0.000000 & 0.000000 & 1.295214 \\
\hline & $\mathrm{C}$ & 0.000000 & 1.121689 & -0.647607 \\
\hline & $\mathrm{N}$ & 0.000000 & 0.000000 & -1.379413 \\
\hline & $\mathrm{N}$ & 0.000000 & 1.194607 & 0.689706 \\
\hline \multirow[t]{3}{*}{ s-Tetrazine $\left(\mathrm{D}_{2 h}\right)$} & $\mathrm{H}$ & 0.000000 & 0.000000 & -2.345244 \\
\hline & $\mathrm{C}$ & 0.000000 & 0.000000 & 1.263568 \\
\hline & $\mathrm{N}$ & 0.000000 & 1.201393 & 0.665348 \\
\hline \multirow[t]{3}{*}{ Formaldehyde $\left(\mathrm{C}_{2 v}\right)$} & $\mathrm{H}$ & 0.000000 & 0.936522 & -1.194669 \\
\hline & $\mathrm{C}$ & 0.000000 & 0.000000 & -0.611070 \\
\hline & $\mathrm{O}$ & 0.000000 & 0.000000 & 0.601026 \\
\hline \multirow[t]{5}{*}{ Acetone $\left(\mathrm{C}_{2 v}\right)$} & $\mathrm{H}$ & 0.000000 & 2.146422 & -0.027075 \\
\hline & $\mathrm{H}$ & -0.882224 & 1.327228 & -1.347775 \\
\hline & $\mathrm{C}$ & 0.000000 & 0.000000 & 0.101073 \\
\hline & $\mathrm{C}$ & 0.000000 & 1.290335 & -0.700873 \\
\hline & $\mathrm{O}$ & 0.000000 & 0.000000 & 1.318967 \\
\hline
\end{tabular}




\begin{tabular}{|c|c|c|c|c|}
\hline Molecule (symmetry) & Atom & $\mathrm{X}$ & $\mathrm{Y}$ & $\mathrm{Z}$ \\
\hline \multirow{4}{*}{ p-Benzoquinone $\left(\mathrm{D}_{2 h}\right)$} & $\mathrm{H}$ & 0.000000 & 2.186299 & 1.257330 \\
\hline & $\mathrm{C}$ & 0.000000 & 0.000000 & 1.447657 \\
\hline & $\mathrm{C}$ & 0.000000 & 1.272483 & 0.672789 \\
\hline & $\mathrm{O}$ & 0.000000 & 0.000000 & 2.672016 \\
\hline \multirow[t]{6}{*}{ Formamide $\left(\mathrm{C}_{s}\right)$} & $\mathrm{H}$ & -0.035245 & -1.510395 & 0.000000 \\
\hline & $\mathrm{H}$ & 1.985297 & -0.387754 & 0.000000 \\
\hline & $\mathrm{H}$ & 1.220829 & 1.174175 & 0.000000 \\
\hline & $\mathrm{C}$ & -0.086417 & -0.408385 & 0.000000 \\
\hline & $\mathrm{O}$ & -1.137024 & 0.203772 & 0.000000 \\
\hline & $\mathrm{N}$ & 1.148803 & 0.169288 & 0.000000 \\
\hline \multirow[t]{8}{*}{ Acetamide $\left(\mathrm{C}_{s}\right)$} & $\mathrm{H}$ & -0.429500 & 1.963037 & 0.000000 \\
\hline & $\mathrm{H}$ & -1.825424 & 0.919709 & 0.000000 \\
\hline & $\mathrm{H}$ & 1.968854 & -0.768041 & 0.000000 \\
\hline & $\mathrm{H}$ & 1.731392 & 0.760691 & 0.885868 \\
\hline & $\mathrm{C}$ & -0.048142 & -0.085394 & 0.000000 \\
\hline & $\mathrm{C}$ & 1.447136 & 0.186847 & 0.000000 \\
\hline & $\mathrm{O}$ & -0.530756 & -1.207321 & 0.000000 \\
\hline & $\mathrm{N}$ & -0.826744 & 1.039291 & 0.000000 \\
\hline \multirow[t]{10}{*}{ Propanamide $\left(\mathrm{C}_{s}\right)$} & $\mathrm{H}$ & -1.718718 & 1.621719 & 0.000000 \\
\hline & $\mathrm{H}$ & -2.558968 & 0.106405 & 0.000000 \\
\hline & $\mathrm{H}$ & 0.700516 & 1.446972 & 0.880124 \\
\hline & $\mathrm{H}$ & 2.8946227 & 0.629339 & 0.000000 \\
\hline & $\mathrm{H}$ & 2.0703450 & -0.674316 & -0.880899 \\
\hline & $\mathrm{C}$ & -0.5133681 & -0.079956 & 0.000000 \\
\hline & $\mathrm{C}$ & 0.7402250 & 0.793991 & 0.000000 \\
\hline & $\mathrm{C}$ & 2.0241565 & -0.031604 & 0.000000 \\
\hline & $\mathrm{O}$ & -0.4793028 & -1.300110 & 0.000000 \\
\hline & $\mathrm{N}$ & -1.6935733 & 0.618024 & 0.000000 \\
\hline \multirow[t]{13}{*}{ Cytosine $\left(\mathrm{C}_{s}\right)$} & $\mathrm{H}$ & 2.103205 & 1.409422 & 0.000000 \\
\hline & $\mathrm{H}$ & 0.192912 & 2.827585 & 0.000000 \\
\hline & $\mathrm{H}$ & -2.076127 & 1.733417 & 0.000000 \\
\hline & $\mathrm{H}$ & -3.230237 & -0.441208 & 0.000000 \\
\hline & $\mathrm{H}$ & -2.342275 & -1.940394 & 0.000000 \\
\hline & $\mathrm{C}$ & 0.057348 & 1.753262 & 0.000000 \\
\hline & $\mathrm{C}$ & -1.160876 & 1.158804 & 0.000000 \\
\hline & $\mathrm{C}$ & -1.160455 & -0.287378 & 0.000000 \\
\hline & $\mathrm{C}$ & 1.168040 & -0.430384 & 0.000000 \\
\hline & $\mathrm{O}$ & 2.229243 & -1.026481 & 0.000000 \\
\hline & $\mathrm{N}$ & -0.075930 & -1.033377 & 0.000000 \\
\hline & $\mathrm{N}$ & 1.184394 & 0.990106 & 0.000000 \\
\hline & $\mathrm{N}$ & -2.355274 & -0.933983 & 0.000000 \\
\hline \multirow{14}{*}{ Thymine $\left(\mathrm{C}_{s}\right)$} & $\mathrm{H}$ & -1.808790 & 1.976164 & 0.000000 \\
\hline & $\mathrm{H}$ & -0.960101 & -1.978634 & 0.000000 \\
\hline & $\mathrm{H}$ & 0.472725 & 2.586214 & 0.000000 \\
\hline & $\mathrm{H}$ & 2.845328 & 1.936434 & 0.000000 \\
\hline & $\mathrm{H}$ & 3.138260 & 0.420783 & 0.880445 \\
\hline & $\mathrm{C}$ & -1.629921 & -0.052812 & 0.000000 \\
\hline & $\mathrm{C}$ & 0.757392 & -0.836268 & 0.000000 \\
\hline & $\mathrm{C}$ & 1.185247 & 0.572789 & 0.000000 \\
\hline & $\mathrm{C}$ & 0.232776 & 1.529218 & 0.000000 \\
\hline & $\mathrm{C}$ & 2.660700 & 0.859484 & 0.000000 \\
\hline & $\mathrm{O}$ & -2.818136 & -0.308378 & 0.000000 \\
\hline & $\mathrm{O}$ & 1.510510 & -1.794389 & 0.000000 \\
\hline & $\mathrm{N}$ & -1.122941 & 1.236814 & 0.000000 \\
\hline & $\mathrm{N}$ & -0.634602 & -1.019928 & 0.000000 \\
\hline \multirow[t]{3}{*}{ Uracil $\left(\mathrm{C}_{s}\right)$} & $\mathrm{H}$ & -2.017408 & -1.525923 & 0.000000 \\
\hline & $\mathrm{H}$ & -0.037071 & 2.000601 & 0.000000 \\
\hline & $\mathrm{H}$ & 2.186816 & -1.597809 & 0.000000 \\
\hline
\end{tabular}




\begin{tabular}{|c|c|c|c|c|}
\hline Molecule (symmetry) & Atom & $\mathrm{X}$ & $\mathrm{Y}$ & $\mathrm{Z}$ \\
\hline & $\mathrm{H}$ & -0.017760 & -2.789145 & 0.000000 \\
\hline & $\mathrm{C}$ & -1.239995 & 0.355737 & 0.000000 \\
\hline & $\mathrm{C}$ & 1.277990 & 0.401032 & 0.000000 \\
\hline & $\mathrm{C}$ & 1.247659 & -1.064625 & 0.000000 \\
\hline & $\mathrm{C}$ & 0.063207 & -1.709410 & 0.000000 \\
\hline & $\mathrm{O}$ & -2.304131 & 0.941040 & 0.000000 \\
\hline & $\mathrm{O}$ & 2.276936 & 1.095016 & 0.000000 \\
\hline & $\mathrm{N}$ & -1.138733 & -1.030829 & 0.000000 \\
\hline & $\mathrm{N}$ & -0.005322 & 0.988101 & 0.000000 \\
\hline \multirow[t]{15}{*}{ Adenine $\left(\mathrm{C}_{s}\right)$} & $\mathrm{H}$ & 2.011864 & -2.505708 & 0.000000 \\
\hline & $\mathrm{H}$ & 1.265984 & 2.708859 & 0.000000 \\
\hline & $\mathrm{H}$ & 2.822411 & 1.913573 & 0.000000 \\
\hline & $\mathrm{H}$ & -2.721643 & -1.389953 & 0.000000 \\
\hline & $\mathrm{H}$ & -3.312695 & 1.099409 & 0.000000 \\
\hline & $\mathrm{C}$ & 1.351251 & -1.644974 & 0.000000 \\
\hline & $\mathrm{C}$ & 1.214246 & 0.657399 & 0.000000 \\
\hline & $\mathrm{C}$ & -0.188214 & 0.522517 & 0.000000 \\
\hline & $\mathrm{C}$ & -0.677566 & -0.781382 & 0.000000 \\
\hline & $\mathrm{C}$ & -2.302961 & 0.717203 & 0.000000 \\
\hline & $\mathrm{N}$ & 0.042556 & -1.915431 & 0.000000 \\
\hline & $\mathrm{N}$ & 1.976462 & -0.449014 & 0.000000 \\
\hline & $\mathrm{N}$ & -2.048158 & -0.639855 & 0.000000 \\
\hline & $\mathrm{N}$ & -1.219795 & 1.457921 & 0.000000 \\
\hline & $\mathrm{N}$ & 1.817944 & 1.868950 & 0.000000 \\
\hline
\end{tabular}

